\section{Microchimica Acta: Quo vadis?}

\author{
Alberto Escarpa ${ }^{1}$ (D) $\cdot$ Mamantos Prodromidis $^{2}$ (D)
}

Published online: 8 January 2020

(C) Springer-Verlag GmbH Austria, part of Springer Nature 2020

Honored and aware of our responsibilities, we want to share with you our great pleasure to be the new Editors-in-Chief (EiC) of Microchimica Acta (MCA). Founded back in 1937 under the name Mikrochemie by Nobel laureate Fritz Pregl, MCA has become a leading journal in analytical chemistry, with a clear focus on (bio)analytical methods based on the use of advanced micro/nano-materials and -structures. It is our firm intention to tackle the challenge of maintaining the journal's quality established by our predecessor, Prof. Otto Wolfbeis.

MCA has established itself as a privileged forum in cuttingedge research, and its multi-disciplinarity has been the driving force of MCA and is key to its success. As stated in the MCA Aims \& Scope, this journal is a forum that shares new discoveries, methods, and instrumentation related to the use of micro/nano-structured materials not only in the fascinating landscape of fundamental analytical and bioanalytical sciences and in material sciences, but also in applications such as in clinical, food, industrial and environmental chemistry. Drawing a 'chemical-timeline' between 2011 (the 'Year of Chemistry') and 2019 (the 'International Year of the Periodic Table of Chemical Elements'), the metrics of MCA have registered a significant leap forward; its impact factor increased from 3.0 (in 2011) to 5.5 (in 2018), with a five year impact factor of 5.1 (in 2018). The journal receives around 2200 submissions per year. Regardless of the metrics, MCA has been recognized by our authors as a highly reputed analytical journal.

\section{Alberto Escarpa}

alberto.escarpa@uah.es

Mamantos Prodromidis mprodrom@uoi.gr

1 Department of Analytical Chemistry, Physical Chemistry and Chemical Engineering. Chemical Research Institute "Andrés M. del Río”, University of Alcalá, 28871 Alcalá de Henares, Madrid, Spain

2 Department of Chemistry, University of Ioannina, 45 110 Ioannina, Greece
We feel highly honored to be the new Editors-in-Chief (EiC) and we are ready to serve the analytical community. In our new function, we are asking ourselves: Quo vadis, MCA? Given the success in the past years, the answer is obvious: As we are already on the right path, let us continue in this way! MCA's scope is clear: a combination of analytical sciences and of micro- and nano-materials. We are therefore encouraging those who have published in MCA before to continue to submit their manuscripts, and those who have not yet contributed, to consider MCA as a channel for publishing their work. Wide attention is certainly warranted given the worldwide interest in technologies based on the use of nanomaterials, including analytical methods. Manuscripts describing the preparation and use of advanced nanomaterials such as (noble) metal nanoparticles, quantum dots, spectral shifters, carbon nanomaterials, 2D materials, nanocomposites, nanosorbents, nanocatalysts, or enzyme mimics are highly welcome at MCA. In any case, novelty has to be demonstrated, and usually a good rationale should be given as to why the (nano)material is used.

Functional micro/nano-materials have been shown to be useful in a plethora of analytical methods. Sensor construction, sensing strategies, separations and extractions, nano-imprints, composites, frameworks, logic gates, lab-on-achip approaches and, more recently, dynamic micro/ nano-machines, motors and robots, all take advantage of these materials. Their use results in advanced methods in electrochemical and optical assays, in biosensors, in preconcentration and separation science, in mass spectrometry and in lateral flow assays, to give some examples. In the majority of cases, the nanomaterial is modified such that recognition becomes possible, for example by using organic receptors, chelators, imprints, oligonucleotide probes, antibodies or aptamers.

Such new methods have been applied to the analysis of clinical, biological, environmental and food samples, including in-vivo applications, cellular imaging and DNA assays. It is already obvious, after only some 20 years of more intense research in the field, that nanotechnology has made a 
triumphal impact on modern analytical sciences, and in society in general. We are convinced that MCA will continue to be a stimulus for further progress and success.

As stated above, MCA receives more than 2200 manuscripts per year. Given these numbers, and due to the retirement of Professor Otto Wolbeis as EiC, the editorial team has been redefined and, as of January 2020, it is made up of, apart from ourselves, a group of Senior Associate Editors (SAE) and Associate Editors (AE) with expertise in the relevant areas of analytical sciences based on nanomaterials.

Manuscripts are reviewed by at least two expert reviewers. In parallel, MCA has established a comprehensive internal reviewing system that aims to a) warrant the scientific and formal quality of the manuscripts in order to avoid certain scientific and formal deficiencies that may have been overlooked by reviewers and b) assist mainly our young authors to prepare top-quality manuscripts. The team of AE will take care of the internal review. We believe that this highly qualified editorial team and the stringent reviewing process will warrant that excellent work will be published in MCA also in future.

Concluding this letter, we would like to thank Prof. Otto Wolfbeis for the excellent work he has done all these years, the (Senior) Associate Editors and the members of the Advisory Editorial Board for their continuous support in handling manuscripts and making good decisions, the Managing Editors and finally, Dr. Steffen Pauly of Springer Nature for his continuous support and valuable cooperation.

Thank you all very much!

We are glad to invite you to submit your best work to Microchimica Acta.
Alberto Escarpa
Editor-in-Chief, Microchimica Acta
Mamas Prodromidis
Editor-in-Chief, Microchimica Acta 